\title{
Phase-Slip Processes in Superconducting Films: AC Josephson Effect and New Phase Transition
}

\author{
V.M. Dmitrievi, ${ }^{a, b}$, I.V. Zolochevskit ${ }^{a}$, E.V. Bezuglyi $^{a}$ \\ AND D.S. KONDRASHEV ${ }^{a}$ \\ ${ }^{a}$ B. Verkin Institute for Low Temperature Physics and Engineering \\ National Academy of Sciences of Ukraine, 61103 Kharkov, Ukraine \\ ${ }^{b}$ International Laboratory of High Magnetic Fields and Low Temperatures \\ Gajowicka 95, 53-421 Wrocław, Poland
}

\begin{abstract}
The resistive direct current state in wide thin superconducting films as the phase transition from superconducting to normal state is investigated in the absence of external magnetic field. We showed that the states of a wide film with increasing transport current appear in the following order: the superconducting state; the resistive vortex state; the critical state; the new vortex-free resistive state with phase-slip lines; the normal state. We detected the Shapiro current steps on the current-voltage characteristic of the wide film, which arise due to interaction of intrinsic Josephson radiation of phase-slip lines with external microwave irradiation.
\end{abstract}

PACS numbers: 74.25.Fy, 74.25.Nf, 74.50.+r

\section{Introduction}

The resistive current state in the superconducting thin films is nonstationary and nonequilibrium due to appearance of so-called phase-slip centers (PSCs) and phase-slip lines (PSLs). There is a big difference between the narrow flux-free film and a wide film with vortices.

It is well known that the narrow vortex-free thin film (narrow channel) with a transport current possesses the transition from superconducting to normal state by arising of the phase-slip centers with increasing current. Up to present days the resistive state in a wide film was associated with the vortex motion only. In this investigation we give the evidence that the situation is different.

${ }^{*}$ corresponding author; e-mail: dmitriev@ilt.kharkov.ua 


\section{Experimental results and discussion}

The objects of the study were thin $\left(d \ll \xi(T), \lambda_{\perp}(T)\right)$ tin films prepared by the technique described in [1]. (Here $\xi(T)$ is the coherence length, $\lambda_{\perp}(T)$ is magnetic field penetration depth into the film.) During the measurement of the current-voltage characteristics (IVCs) the samples were placed in a double shield of annealed permalloy. The parameters of some films studied are presented in Table.

TABLE

Parameters of the film samples. Here $L$ is the length, $w$ - the width, and $d$ - the thickness of the sample; $l_{i}$ is the electron mean free path.

\begin{tabular}{c|c|c|c|c|c|c|c}
\hline \hline Sample & $\begin{array}{c}L \\
{[\mu \mathrm{m}]}\end{array}$ & $\begin{array}{c}w \\
{[\mu \mathrm{m}]}\end{array}$ & $\begin{array}{c}d \\
{[\mathrm{~nm}]}\end{array}$ & $\begin{array}{c}R_{4.2} \\
{[\Omega]}\end{array}$ & $\begin{array}{c}T_{\mathrm{c}} \\
{[\mathrm{K}]}\end{array}$ & $\begin{array}{c}l_{i} \\
{[\mathrm{~nm}]}\end{array}$ & $\begin{array}{c}R_{300} \\
{[\Omega]}\end{array}$ \\
\hline SnW5 & 92 & 42 & 120 & 0.14 & 3.789 & 145 & 2.270 \\
SnW10 & 88 & 7.3 & 181 & 0.487 & 3.809 & 169 & 9.156 \\
SnW12 & 90 & 18 & 332 & 0.038 & 3.836 & 466 & 1.880
\end{tabular}

The IVC of one of the samples, typical of the high-quality films, is shown in Fig. 1. The direct current-induced resistive state of the film, due to motion of the lattice of intrinsic Abrikosov vortices, exists in the current interval $I_{\mathrm{c}} \leq I \leq I_{\mathrm{m}}$ (the vortex part of the IVC). A theory of such resistive state due to vortex motion was proposed by Aslamazov and Lempitskiy (AL) in [2] and gives the value of $I_{\mathrm{c}}^{\mathrm{AL}}(T)$, which is the highest of the theoretically predicted critical currents for the entry of Abrikosov vortices into the film

$$
I_{\mathrm{c}}^{\mathrm{AL}}(T)=1.5 I_{\mathrm{c}}^{\mathrm{GL}}(0)\left(\pi \lambda_{\perp}(0) / w\right)^{1 / 2}\left(1-T / T_{\mathrm{c}}\right) .
$$

The expression for the maximum current $I_{\mathrm{m}}(T)$ for the existence of the uniform flux flow is obtained in [2]:

$$
I_{\mathrm{m}}(T)=C I_{\mathrm{c}}^{\mathrm{GL}}(T) \ln ^{-1 / 2}\left(2 w / \lambda_{\perp}(T)\right) .
$$

Here $C$ is a fitting numerical coefficient. At $I=I_{\mathrm{m}}$, the current density becomes equal to the critical one not only at the edges, where vortices are nucleated, but also in the middle of the film $[2,3]$. By the present investigation we confirm that at $I>I_{\mathrm{m}}(T)$ the resistive vortex state becomes unstable, and the wide film undergoes a jump-like transition to the new resistive state due to the appearance of a phase-slip line [1] already in the absence of a vortex structure.

A natural question arising here is whether a fundamental difference exists between PSCs and PSLs? We cannot give an unambiguous answer to this question. Indeed, it does not exist because both the PSC and the PSL are the products of a mechanism of phase-slip of the order parameter, but at the same time, it does, because the initial situation for the PSC and PSL are fundamentally different. 


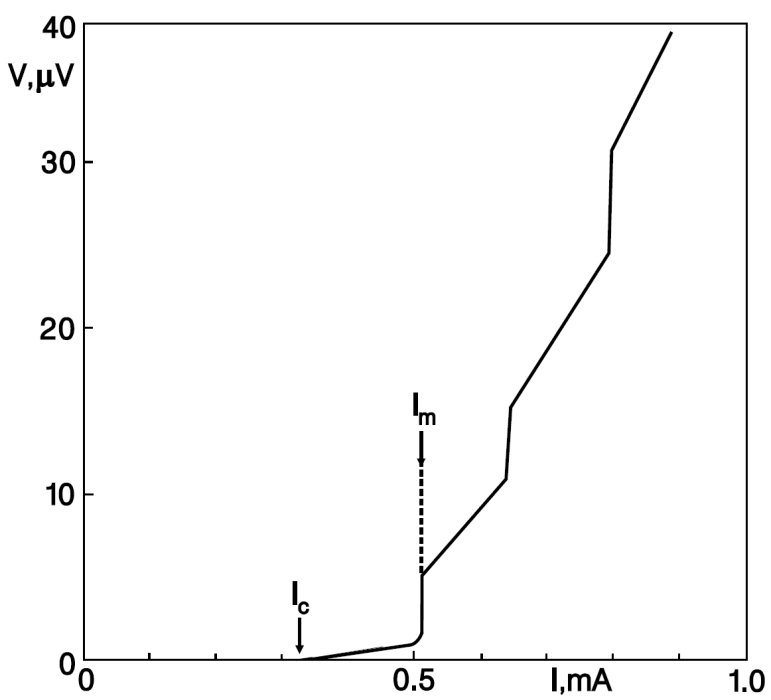

Fig. 1. Typical $I-V$ characteristic of a wide $\left(w \gg \xi(T), \lambda_{\perp}(T)\right)$ superconducting film (sample SnW12) at a temperature $T=3.798 \mathrm{~K} . I_{\mathrm{m}}$ is the maximum current of the existence of the vortex state, $I_{\mathrm{c}}$ is the critical current of the wide film.

The initial state for the PSC is a purely superconducting state with the Ginzburg-Landau critical current $I_{\mathrm{c}}^{\mathrm{GL}}(T)$. In narrow films a system of PSCs arises in the transition region $I_{\mathrm{c}}^{\mathrm{GL}}<I<I_{\mathrm{c} 2}$ between the purely superconducting and the normal states, the latter is reached at $I \geq I_{\mathrm{c} 2}$. The initial state for the PSLs is the vortex instability of the state with a nearly uniform but nevertheless specific distribution of current over the width of the film [2].

We have shown that the states of a wide film with increasing transport current appear in the following order: the superconducting state at the current $I<I_{\mathrm{c}}$; the resistive vortex state at $I_{\mathrm{c}}<I<I_{\mathrm{m}}$; the critical state due to the onset of instability of the steady pattern of viscous motion of the vortices at $I=I_{\mathrm{m}}$; a vortex-free resistive state with phase-slip lines for the current $I$ larger than the instability current $I_{\mathrm{m}}$, but smaller than the upper critical current $I_{\mathrm{c} 2}$; and the normal state at a current higher than the upper critical current $I_{\mathrm{c} 2}\left(I>I_{\mathrm{c} 2} \gg I_{\mathrm{m}}\right)$. Therefore, there is a new, vortex-free state within the current phase transition from the superconducting to the normal state.

The PSL is a line transverse to the film, at which the order parameter executes in-phase oscillations at the Josephson frequency, vanishing at certain times simultaneously along the whole line transverse to the film. These oscillations are a source of nonequilibrium quasiparticles, the diffusion of which into the superconducting region determines the longitudinal size of the PSL. This state is analogous to the state of a narrow vortex-free superconducting channel with a PSCs. As soon as we suggest that phase slip processes are similar in a narrow channel and 
in a wide film, it would be natural to use the experience obtained when investigating the ac Josephson properties of PSC, considering the PSL as a source of the Josephson radiation.
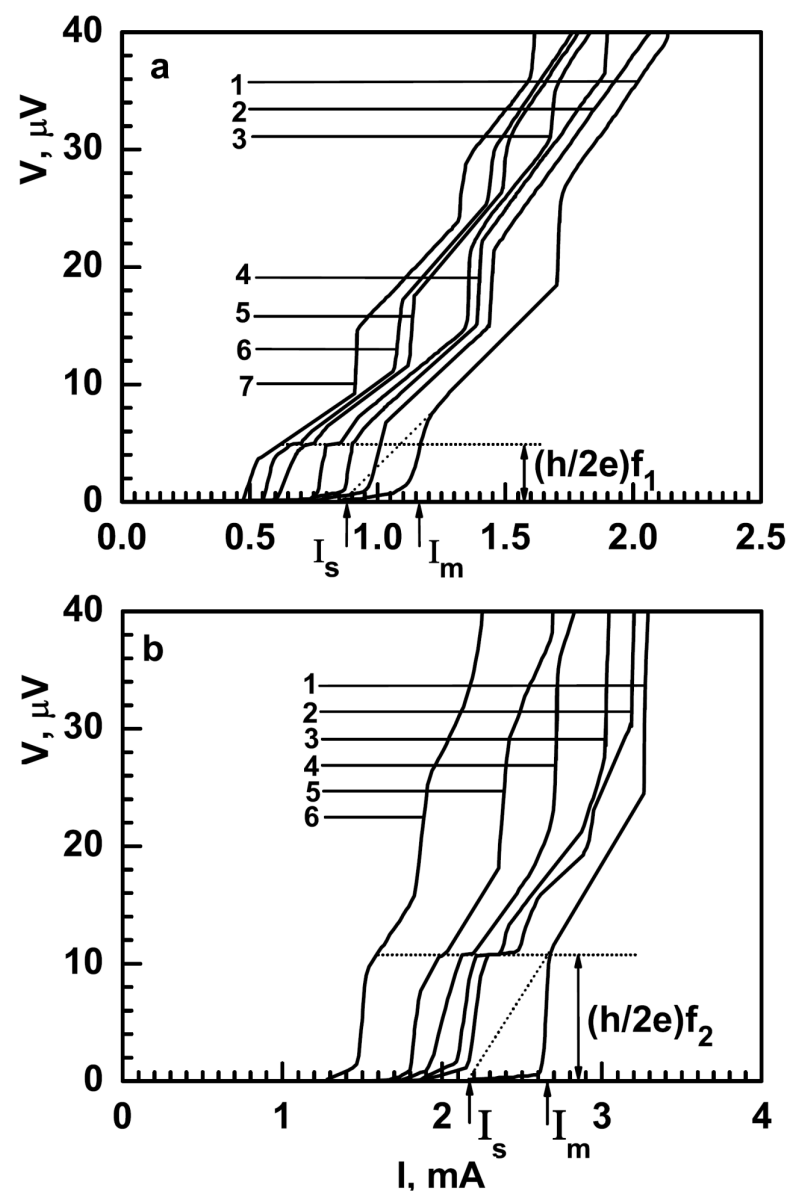

Fig. 2. A family of IVCs for the films SnW12 at $T=3.807 \mathrm{~K}$ and $f_{1}=2476 \mathrm{MHz}$ (a) and SnW5 at $T=3.744 \mathrm{~K}$ and $f_{2}=5500 \mathrm{MHz}$ (b). For curve 1, the applied irradiation power equals zero; for the others it increases with the IVC number.

To obtain the evidence of the Josephson properties of the PSL, for the first time we have detected the so-called Shapiro current steps [4] on the IVC of the wide film. These steps are the result of the interaction between the intrinsic Josephson radiation of PSL and the applied external electromagnetic field. Families of the IVCs for the SnW12 and SnW5 samples, (a) and (b), respectively, measured at different irradiation frequency and power, are presented in Fig. 2. We note that the inequality $w / \lambda_{\perp}(T) \geq 20$ is fulfilled for both films and their IVCs contain initial resistive regions caused by the vortex motion, i.e., both films can be unambiguously 
referred to as wide ones. The IVCs of the films on initiation of PSLs have the same shape as the IVC of a narrow channel on initiation of PSCs: they reveal abrupt voltage steps, cut-off current $I_{\mathrm{S}}$ at zero voltage, excess current at high voltages, and the sample resistance changes by a multiple: $R=n R_{\mathrm{d} 1}$, where $R_{\mathrm{d} 1}$ is the dynamic resistance of a IVC linear portion corresponding to the first PSL and $n$ is the number of PSLs in the film. Under the microwave irradiation, current steps in the IVCs occur at fixed voltage, proportional to the frequency $f[4]$ :

$$
V=\frac{n h}{2 e} f \quad(n=1,2, \ldots)
$$

which are known to result from the interaction between the applied microwave field and the intrinsic ac Josephson current.

Due to the temperature dependence of $\lambda_{\perp}(T)$ it is possible to distinguish the temperature interval $T_{\text {cros }}<T<T_{\mathrm{c}}$ in a close vicinity of $T_{\mathrm{c}}$, where $w<\lambda_{\perp}(T)$. At $T<T_{\text {cros }}, w>\lambda_{\perp}(T)$. Thus, $T_{\text {cros }}$ separates the regions of "narrow" and "wide" regimes in the same sample. This gives the possibility to observe voltage steps caused by the formation of PSCs and PSLs at $T>T_{\text {cros }}$ and $T<T_{\text {cros }}$ accordingly, which was detected in our experiments.

\section{Conclusion}

We have experimentally determined for the first time the values and the temperature dependence of the critical current $I_{\mathrm{c}}^{\mathrm{AL}}(T)$ and the maximum current for the existence of vortex resistance (the instability current) $I_{\mathrm{m}}(T)$ predicted by the theory of Aslamazov and Lempitskiy [2] for wide superconducting films. We have shown that for $I \geq I_{\mathrm{m}}(T)$ a system of lines of slipping of the order parameter phase (PSLs) develops, which determines the film resistance in the new vortex-free final stage of its transition from the superconducting to the normal state with increasing transport current.

The current steps resulted from the interaction between the intrinsic Josephson radiation of phase-slip line and the applied electromagnetic field were first observed in the current-voltage characteristics of deliberately wide superconducting films, whose transition to the resistive state with phase slip lines is preceded by creation of a vortex state.

We have first experimentally observed PSCs and PSLs in the same superconducting tin films with known parameters in the temperature intervals corresponding to the mechanisms of their formation and existence.

\section{References}

[1] V.M. Dmitriev, I.V. Zolochevskii, Supercond. Sci. Technol. 19, 342 (2006).

[2] L.G. Aslamazov, S.V. Lempitskiy, Sov. Phys. JETP 57, 1291 (1983).

[3] A.G. Sivakov, A.P. Zhuravel', O.G. Turutanov, I.M. Dmitrenko, Czech. J. Phys. 46, 877 (1996).

[4] S. Shapiro, Phys. Rev. Lett. 11, 80 (1963). 